\title{
Polarizer-free polarimetric image sensor through anisotropic two-dimensional GeSe
}

\author{
Xiaoting Wang ${ }^{1 \dagger}$, Fang Zhong ${ }^{2 \dagger}$, Jun Kang ${ }^{3}$, Can Liu ${ }^{4}$, Ming Lei ${ }^{5}$, Longfei Pan ${ }^{1}$, Hailu Wang ${ }^{2}$, \\ Fang Wang ${ }^{2}$, Ziqi Zhou ${ }^{1}$, Yu Cui ${ }^{1}$, Kaihui Liu $^{4}$, Jianlu Wang ${ }^{2}$, Guozhen Shen ${ }^{1}$, Chongxin Shan ${ }^{6}$, \\ Jingbo $\mathrm{Li}^{7}$, Weida $\mathrm{Hu}^{2^{*}}$ and Zhongming $\mathrm{Wei}^{{ }^{*}}$
}

\begin{abstract}
Light polarization could provide critical visual information (e.g., surface roughness, geometry, or orientation) of the imaged objects beyond prevailing signals of intensity and wavelength. The polarization imaging technology thus has a large potential in broad fields such as object detection. However, intricate polarization coding is often required in these fields, and the existing complicated lensed system and polarizers have limited the miniaturization capabilities of the integrated imaging sensor. In this study, we demonstrate the utilization of two-dimensional (2D) in-plane anisotropic $\alpha$-GeSe semiconductor to realize the polarizer-free polarization-sensitive visible/near-infrared (VIS-NIR) photodetector/imager. As the key part of the sensor system, this prototype $\mathrm{Au} / \mathrm{GeSe} / \mathrm{Au}$ photodetector exhibits impressive performances in terms of high sensitivity, broad spectral response, and fast-speed operation $\left(\sim 10^{3} \mathrm{~A} \mathrm{~W}^{-1}, 400-1050 \mathrm{~nm}\right.$, and 22.7/49.5 $\mu \mathrm{s})$. Further, this device demonstrates unique polarization sensitivity in the spectral range of $690-1050 \mathrm{~nm}$ and broadband absorption of light polarized preferentially in the $y$-direction, as predicted by the analysis of optical transition behavior in $\alpha-G e S e$. Then we have successfully incorporated the $2 \mathrm{D}$ GeSe device into an imaging system for the polarization imaging and captured the polarization information of the radiant target with a high contrast ratio of 3.45 at $808 \mathrm{~nm}$ (NIR band). This proposed imager reveals the ability to sense dual-band polarization signals in the scene without
\end{abstract}

polarizers and paves the way for polarimetric imaging sensor arrays for advanced applications.

Keywords: GeSe, 2D anisotropic semiconductors, polarization sensing detectors, broadband polarimetric imaging

\section{INTRODUCTION}

Infrared polarization imaging is a powerful sensing technology that can provide richer sets of information about an object in the scene such as intensity, spectral, and polarization [1-5]. Polarization information finds surface properties of an object, such as the polarization state of the light reflected/scattered at the surface $[6,7]$. This effectively enhances the resolving power of visionbased detection systems and could be utilized in many specialized applications such as target tracking, remote sensing [8,9], and computer vision [10]. However, detectors used for these applications typically require the use of external elements such as the prepositive polarizer array for intricate polarization coding [11], which increases the size and complexity of these systems and imposes a limit on the resolution for imaging purpose [12-14]. As such it is important to develop the polarization sensing detector capable of sensing both the intensity and the polarization of an incoming light. Discovering further entirely novel photoelectric devices as

\footnotetext{
${ }^{1}$ State Key Laboratory of Superlattices and Microstructures, Institute of Semiconductors, Chinese Academy of Sciences \& Center of Materials Science and Optoelectronics Engineering, University of Chinese Academy of Sciences, Beijing 100083, China

${ }^{2}$ State Key Laboratory of Infrared Physics, Shanghai Institute of Technical Physics, Chinese Academy of Sciences, Shanghai 200083, China

${ }^{3}$ Beijing Computational Science Research Center, Beijing 100193, China

${ }^{4}$ State Key Laboratory for Mesoscopic Physics, School of Physics, Peking University, Beijing 100871, China

${ }^{5}$ State Key Laboratory of Information Photonics and Optical Communications, School of Science, Beijing University of Posts and Telecommunications, Beijing 100876, China

${ }^{6}$ Henan Key Laboratory of Diamond Optoelectronic Materials and Devices, School of Physics and Engineering, Zhengzhou University, Zhengzhou 450001, China

${ }^{7}$ Institute of Semiconductors, South China Normal University, Guangzhou 510631, China

These two authors contributed equally to this work.

* Corresponding authors (emails: wdhu@mail.sitp.ac.cn (Hu W); zmwei@semi.ac.cn (Wei Z))
} 
micro-polarizers is of great benefit to simplify the current polarization imaging system.

The emerging two-dimensional (2D) materials have shown great potential for use in future polarizationresolved photodetectors, largely from unique physical, transport properties and somewhile intrinsic in-plane anisotropy on the atomic scale. Together with high specific surface area/absorption efficiency/mobility and facile integration, some 2D materials such as graphene and $\mathrm{WSe}_{2}$, have been proposed as promising building blocks for imaging in a wide spectral range and compact sensing systems [15-20]. Except for the isotropic optical behavior of high-symmetry materials, intrinsic anisotropy properties in low-symmetry systems could be introduced for expanding the scope of optoelectronic applications [2123]. Also, the in-plane anisotropy-related practical image sensing applications of $2 \mathrm{D}$ materials have not yet been accessed. Although previous studies have demonstrated the linear dichroic photodetector using black phosphorus (BP) [24], the instability under ambient conditions has been an inherent barrier to its effective use in imaging systems. Then BP analogues-selenide germanium (GeSe)-has received plenty of attention in the study of optoelectronic devices $[25,26]$. This interest is partially based on the highly anisotropic orthorhombic crystal structure (space group Pcmn- $D_{2 h}{ }^{16}$ ) [27]. In addition, GeSe shows its merits such as large absorption coefficient [28], narrow optical bandgap (1.1-1.2 eV), as well as easy preparation [29], and environmental stability.

Here we demonstrate that the physical properties of phosphorene-like $\alpha$-GeSe enable polarization-related photodetectors and polarizer-free imaging systems operating in the visible/near-infrared (VIS-NIR) spectral regime. The prototype Au-GeSe-Au photodetector exhibits impressive performances in terms of high sensitivity, broad spectral response, and fast-speed operation, which can be leveraged for the realization of chip-scale sensors and imagers. Further, this device demonstrates unique polarization sensitivity in the spectral range of $690-1050 \mathrm{~nm}$ and broadband absorption of light polarized preferentially in the $y$-direction, as predicted by the analysis of optical transition behavior in $\alpha-G e S e$. In view of the above, a novel image detecting scheme was proposed and high-resolution polarization imaging for the radiating target was obtained via the GeSe-based image sensor. The polarization information was extracted by the optical polarizer, manifesting the contrast ratio of 2.59, $2.63,3.45$ in the VIS (675 and $700 \mathrm{~nm})$ and NIR $(808 \mathrm{~nm})$ spectral bands, respectively. These results demonstrated here open a way towards the exploitation of GeSe in the multi-spectral polarization imaging system or focal plane polarimeters.

\section{EXPERIMENTAL SECTION}

\section{Growth of GeSe crystal}

Single crystals of GeSe were synthesized using the chemical vapor transport (CVT) method. Commercially available GeSe powder (99.99\%, Sigma Aldrich) as the precursor was securely sealed in an evacuated quartz tube under high vacuum $\left(10^{-5}\right.$ Torr $)$ and subsequently mounted into the tube furnace. Then the CVT reaction was initiated with the $T_{1}$ zone kept at $640^{\circ} \mathrm{C}$ and $T_{2}$ zone at $450^{\circ} \mathrm{C}$ in $20 \mathrm{~cm}$ for $10 \mathrm{~h}$. Next, the operative temperatures of the two zones were permutated $\left(T_{1}=450^{\circ} \mathrm{C}\right.$, $T_{2}=640^{\circ} \mathrm{C}$ ) and worked for $10 \mathrm{~h}$. Afterward, the furnace was cooled down to $300^{\circ} \mathrm{C}$ in two days, and the black shining GeSe crystals in sizes up to $60-100 \mathrm{~mm}$ were found in the cold zone $\left(T_{2}\right)$ of the ampule. And the thinlayer GeSe crystals attached on the $(300 \mathrm{~nm}) \mathrm{SiO}_{2} / \mathrm{Si}$ substrates could be acquired by exfoliation from the bulk phases of layered crystals.

\section{Optical measurements and characterization}

In experiments about polarization, the laser beam passed through a linear polarizer, a half-wave plate (HWP) and was then focused on the GeSe channel using a $\times 20 \mathrm{mi}-$ croscope objective. For the spectral response, the grating spectrometer was used to select the desired wavelength from the supercontinum white-light laser source (YSL SC-HP). The scanning photocurrent mapping was investigated by a scanning Galvo System (Thorlabs GVS212) with the modulated laser beam. The modulated photocurrent signals were amplified and extracted from a lock-in technique (Signal Recovery model 7270). The polarization-resolved imaging detection was carried out by a home-made imaging system, and the $2 \mathrm{D}$ GeSe device was used as the photosensitive unit in the imaging sensor. For optical spectral analysis, the microscopic spectrophotometer (Jasco MSV-5200) was used with the sampling interval of $0.5 \mathrm{~nm}$, the aperture of $20 \mu \mathrm{m}$ and spectrum range of $400-1200 \mathrm{~nm}$. Thicknesses of GeSe nanoflakes were characterized by an atomic force microscope (AFM, Bruker Dimension Icon scanning probe microscope).

\section{First-principle calculations}

First-principle calculations were done using the projector augmented wave (PAW) method, as implemented in the PWMAT code (a GPU based plane-wave pseudopotential 
code). The room-temperature atomic structure was relaxed using the Perdew-Burke-Enzerhof generalized gradient approximation (GGA) functional, with the convergence threshold of $0.02 \mathrm{eV}^{-1}$ on the forces. The electronic structure and optical properties were then calculated on the optimized structure with the HeydScuseria-Ernzerhof (HSE06) hybrid functions for a higher accuracy. The $k$-mesh for self-consistent calculations was $7 \times 7 \times 1$ and the cutoff energy for the plane-wave basis was fixed at $45 \mathrm{Ry}$.

\section{RESULTS}

Single crystals of a-GeSe were grown directly from the GeSe powders (purity 99.999\%) in a sealed evacuated quartz ampule via the CVT process (see EXPERIMENTAL SECTION). The response time $(\tau)$ and responsivity $\left(R_{\mathrm{ex}}\right)$ are two principal figures of merit for the performance characterization of photodetectors. Here, the rise/fall time $\left(\tau_{\mathrm{r}}, \tau_{\mathrm{f}}\right)$ is usually defined as the time required for the response to rise/fall from $10 \% / 90 \%$ to $90 \% / 10 \%$ of the pulse amplitude [30]. To evaluate the operating speed of the GeSe-based device as in Fig. 1a, the laser was modulated to be $1 \mathrm{~Hz}$ and the photocurrent (PC) generated upon multiple on/off switching of light radiation was collected by a digital oscilloscope with a preamplifier. Through the rising/falling edges related with switch-on/off processes, as in Fig. 1b, this device yielded $\tau_{\mathrm{r}}=22.7 \mu \mathrm{s}$ and $\tau_{\mathrm{f}}=49.5 \mu$ s at the drain-source voltage $\left(V_{\mathrm{ds}}\right)$ of $0.5 \mathrm{~V}$ with $\lambda=830 \mathrm{~nm}$ laser excitation. The photoexcited signal fell fast in about $30 \mu$ s followed by a slow tail, which might be related to the defects/traps or external stress [31]. In spite of this, the speed demonstrated here was fast enough to meet the requirement for imaging or other optical applications. The extrinsic
$R_{\mathrm{ex}}$ is generally expressed as $R_{\mathrm{ex}}=I_{\mathrm{pc}} /\left(P_{\mathrm{in}} A_{\text {device }} / A_{\text {laser }}\right)$ $[32,33]$, where $I_{\mathrm{pc}}=I_{\text {light }}-I_{\text {dark }}$, means the photocurrent signal, $P_{\text {in }}$ denotes the incident light power, $A_{\text {device }}$ is the effective channel area, and $A_{\text {laser }}$ is the laser spot area. We measured the photoresponse of the GeSe in the VIS-NIR range, with typical $R_{\mathrm{ex}}$ data shown in the right $y$-axis of Fig. S1. Herein, $A_{\text {device }}=97.5 \mu \mathrm{m}^{2}, A_{\text {laser }}=0.785 \mathrm{~cm}^{2}$, and $P_{\text {in }}$ is in the micro-watt level with the spot diameter of $1 \mathrm{~cm}$. This device demonstrates the high $R_{\mathrm{ex}}$ along the whole spectral range from VIS to NIR regime at the low light levels $(0.663$ to $4.9 \mu \mathrm{W})$, such as $5920 \mathrm{~A} \mathrm{~W}^{-1}$ at $600 \mathrm{~nm}$ and $850 \mathrm{~A} \mathrm{~W}^{-1}$ at $930 \mathrm{~nm}$, which agrees in a reasonable accuracy with the optical absorption spectra measured in the unpolarized light incidence (left $y$-axis in Fig. S1).

The proof experiment was designed to prove the unpolarized imaging capacity of $2 \mathrm{D}$ GeSe based photodetector. Fig. 1c shows the assembly diagram of functional elements for the image-capturing set-up. The imaging sensor is the focus of the whole system and starts with the aluminium wire-bonding of the device into the 24-pin chip carrier and then the mounting of them on the circuit board that provides the electric connections to the signal processing module. This module provides functions of photosignal acquisition, amplification, quantization as well as photodetector power supply. As with the CCD unit in a digital camera, the GeSe device was used here as the photosensitive unit in the imaging sensor. Fig. S2 contains more details about the electrical connection of the subsystem parts in the signal path. In a typical case, the greyscale plot in the upper right panel of Fig. 1c is compiled of the normalized photosignals for each of photodetection pixels of the $470 \times 1010$ array over the 'Chang'e' pattern, amplified and quantified by the signal
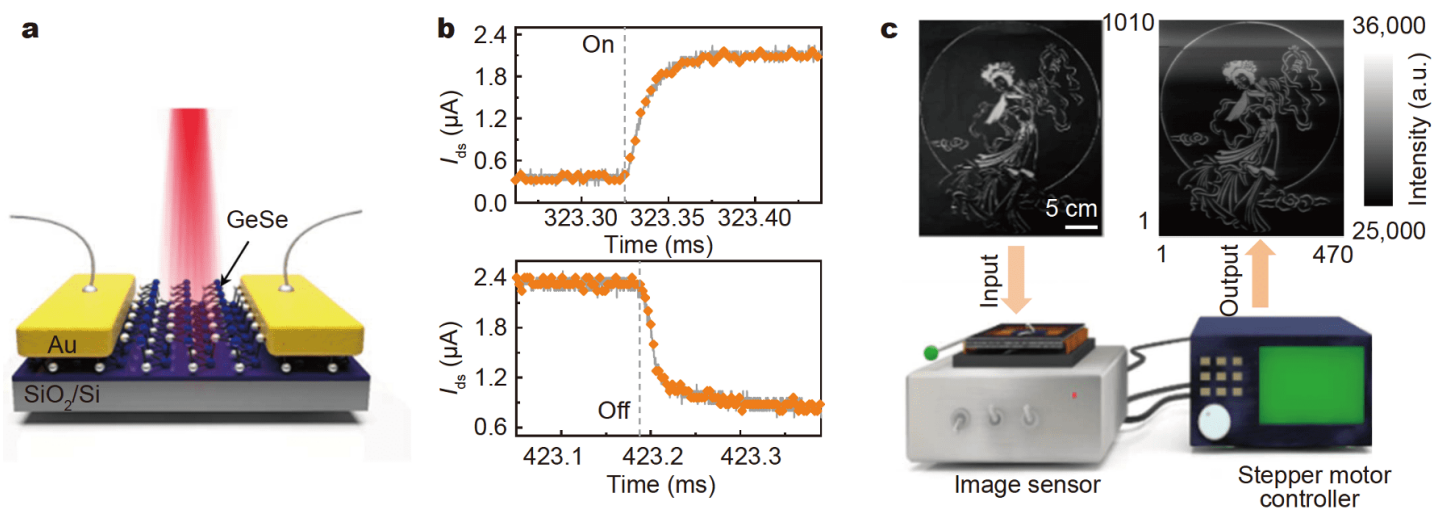

Figure 1 Photoresponse and white-light imaging in 2D GeSe photodetectors. (a) Illustration of a GeSe device used for photoresponse characterization. (b) The rise time ( $22.7 \mu$ s, top) and decay time $\left(\sim 49.5 \mu \mathrm{s}\right.$, bottom) of a typical GeSe device measured at $V_{\mathrm{ds}}=0.5 \mathrm{~V}$ and $V_{\mathrm{g}}=0 \mathrm{~V}$ with laser excitation at $\lambda=830 \mathrm{~nm}$. (c) Schmatic layout of the GeSe-based image scanning system (bottom) and the 'Chang'e' image obtained by this system under excitation of white light (upper right). 
processing circuit [34-36]. For this image, we used the full spectrum of the fluorescent light source to illuminate the shadow mask that was carved with the 'Chang'e' pattern (with minimal line sizes down to $500 \mu \mathrm{m}$ ). The greyscale image measures $\sim 10 \mathrm{~cm}$ in width with a total 470 by 1010 pixel format, which is able to resolve down to $\sim 100 \mu \mathrm{m}$ each pixel. Of note, this GeSe device has provided a stable performance during the data acquisition, so that we have not observed significant fluctuations of the detection signal in these as-measured images. The initial implementation of imaging demonstrates the feasibility of the integration of GeSe-based device with the imaging system.

Key to the actual use of 2D GeSe-based image sensor in the polarimetric imaging system is the excellent ability of sensing the light intensity and polarization, with the absorption efficiency and extinction ratio as critical evaluation parameters. So except for the above light response characteristics, we have performed the polarization sensitive PC studies to understand their dichroic response, with 3D schematic shown in Fig. 2a. The 75.8-nm-thick sample was used for the device. The reflected polarization-resolved optical microscropy (PROM) images under crossed-polarized incident light were first measured by rotating the sample in steps of $15^{\circ}$ (Fig. S3) and helped in determining the crystalline orientations of our as-prepared device (armchair and zigzag) along $0^{\circ}$ or $90^{\circ}$. As an example, the PC scatter plot at the $\lambda_{\mathrm{NIR}}=940 \mathrm{~nm} \mathrm{ex}$ citation, obtained with light polarization at different angles, is shown in Fig. 2c. This plot undergoes maxima and minima showing a two-fold symmetry and is expected to vary with the incident polarization angle $(\theta)$ as the equation of $I_{\mathrm{pc}}(\theta)=I_{\max } \cos ^{2}(\theta-\varphi)+I_{\min } \sin ^{2}(\theta-\varphi)$. Then the fitting result reveals the $I_{\max }$ and $I_{\min }$ equaling to 0.086 and $0.022 \mu \mathrm{A}$, respectively, and the corresponding extinction ratio $r_{\mathrm{e}}$ (expressed as $I_{\max } / I_{\min }$ ) reaches $\sim 3.90$ with incident light polarized along the horizontal/vertical axis. Similar results are found using the 785 and $830 \mathrm{~nm}$ illumination source, as shown in Fig. S4. It could be observed that the GeSe device features high anisotropy, particularly in the NIR region, and this makes GeSe competitive in the polarization application. And their dichroic responses (Fig. 2e) are in a significant similarity to the anisotropic optical absorption (Fig. 2d), and this means that the overall enhancement or loss of dichroic response can be possibly attributed to the variation in optical absorption at particular wavelengths. Another observation from the angle-resolved spectral response is that the PC maximum is attained along the same light polarized direction in the already measured spectral region of 690-1050 nm.
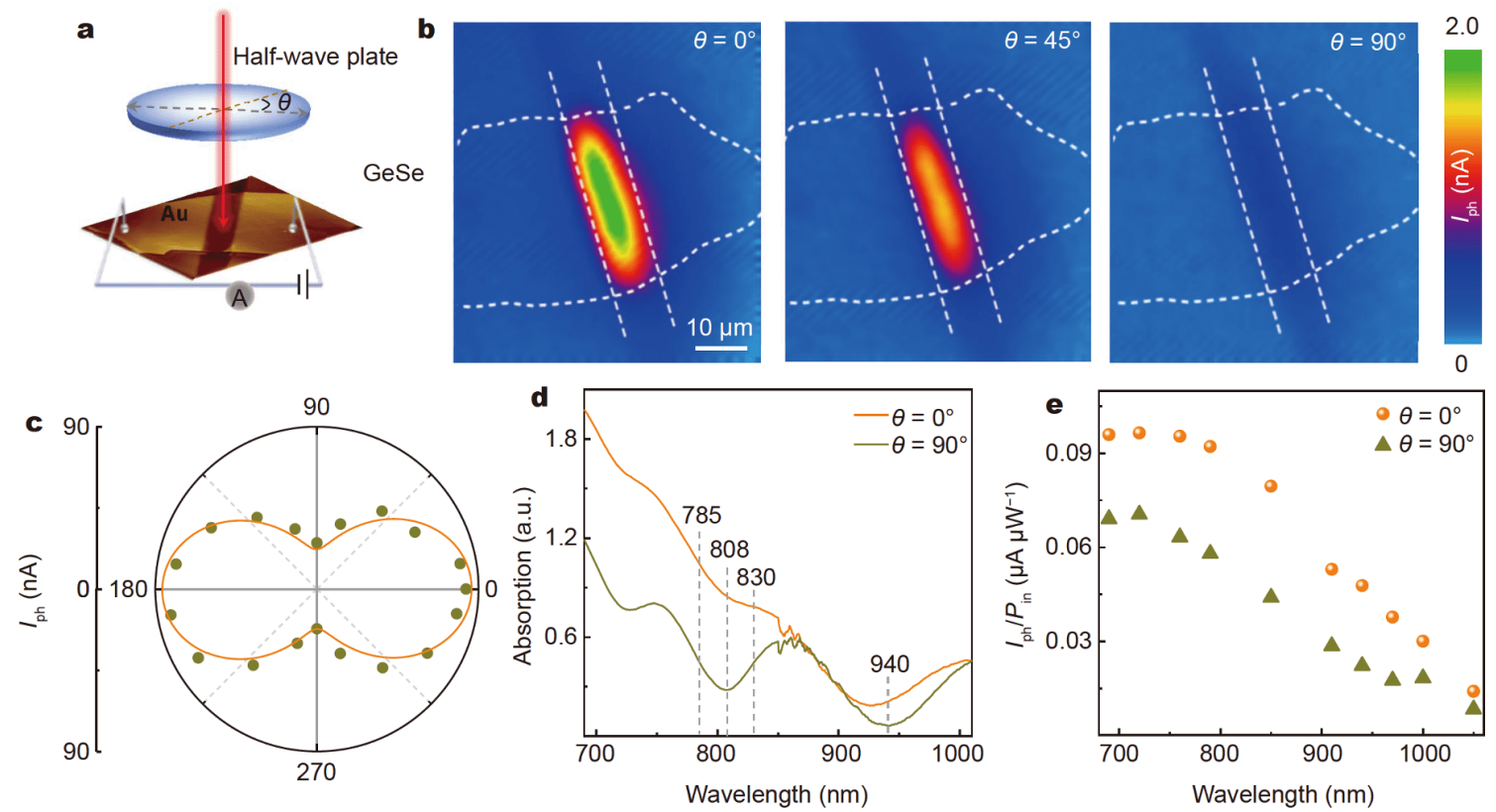

Figure 2 Polarization-sensitivity of GeSe photodetector in the NIR range. (a) 3D schematic diagram of the GeSe polarization-sensitive photodetector. (b) Angle-resolved spatial maps of the GeSe device at $V_{\mathrm{d}}=0.1 \mathrm{~V}$. Areas indicating by white lines are the GeSe sample and Au electrodes. (c) Scatters show the PC at different laser polarizations for the $\lambda_{\mathrm{NIR}}=940 \mathrm{~nm}$ incident laser (with spot diameter of $\sim 15 \mu \mathrm{m}$ and power of $\sim 0.6 \mu \mathrm{W}$ ) at $V_{\mathrm{d}}=1.0 \mathrm{~V}$. (d) Spectrally resolved optical absorption $(400-110 \mathrm{~nm})$. (e) Spectrally resolved photoresponse (690-1050 nm) measured with linearly polarized illumination parallel or perpendicular to the GeSe sample (device). 
High-spatial-resolution mapping allows for locally probing the photoresponse in different regions of the device, and this helps to identify the dominant PC generation mechanism of the photodetector [37]. Fig. 2b presents the spatial maps of the PC intensity with the laser spot scanning across the whole channel of devices. Observed micro-area maps indicate clearly that the photoactive regions are centrally distributed in the GeSe channel rather than the metal-GeSe interface. This could be considered as a typical reveal of the photoconductive effect for PC generation [38]. In the photoconduction mode, the photogenerated electron-hole pairs are driven by applied bias and move in the opposite direction, which directly increases the conductance of this device [39]. In addition to this, the electronic band structures of 4-layer $\mathrm{Au}$ and 4-layer p-GeSe were respectively calculated from the HSE06 functional. As the simplified schematic shown in Fig. S5, the metal work function of $W_{\mathrm{m}}(5.32 \mathrm{eV})$ is greater than that of the semiconductor $W_{\mathrm{s}}(4.87 \mathrm{eV})$. This indicates that the p-type anti-barrier layer (i.e., Ohmic contact) is formed and Au could be well applied as electrode in the GeSe device $[40,41]$. Therefore, it could be inferred theoretically that the prototype Au-GeSe-Au photodetector works in the photoconductive regime, which agrees well with the experimental result of the spatially resolved photocurrent mapping (SPCM).

The optical transition behavior of the material can be used to explain the linear dichroism, anisotropic optical absorption along the $x-y$ (zigzag-armchair) in-plane perpendicular direction and the band structure calculation shown in Fig. 3a, with the bottom of the conduction band (CBM) and top of the valence band (VBM) located at the $\Gamma$ point (direct bandgap). The probability $\left(p_{y}\right)$ of the transition $\left|\varphi_{\mathrm{CBM}}\right\rangle \rightarrow\left|\varphi_{\mathrm{VBM}}\right\rangle$ along the $k$-points path (Fig. 3b) has been calculated from the $p_{y}=\mid\left\langle\varphi_{\text {СвM }}\left|\nabla_{y}\right|\right.$ $\varphi_{\mathrm{VBM}}>\left.\right|^{2} /\left(\left|<\varphi_{\mathrm{CBM}}\right| \nabla_{x}\left|\varphi_{\mathrm{VBM}}>\right|^{2}+\mid\left\langle\varphi_{\mathrm{CBM}}\left|\nabla_{y}\right| \varphi_{\mathrm{VBM}}>\left.\right|^{2}\right)\right.$. Here $\varphi$ denotes the wave function, $\nabla$ is the momentum operator, and $\mid\left\langle\varphi_{\mathrm{CBM}}|\nabla| \varphi_{\mathrm{VBM}}>\left.\right|^{2}\right.$ is the optical transition amplitude. Based on the results in this figure, the detailed optical transition behavior of some critical points such as Г $(1.35 \mathrm{eV}), \mathrm{M}(1.85 \mathrm{eV}), \mathrm{P}(1.92 \mathrm{eV}), \mathrm{K}(2.31 \mathrm{eV})$ have been analyzed. Around the $\Gamma$ point $\left(p_{y}=1.0\right)$, only the absorption of $y$-polarized light occurs across the gap between $\mathrm{Vb}_{\Gamma} \rightarrow \mathrm{Cb}_{\Gamma}$, while that of $x$-polarized light is forbidden. In Fig. $3 c$, we plotted the wavefunctions of CBM and VBM states at $\Gamma$ point and analyzed their symmetrical characteristics to further explore the origin of the polarization. There is a mirror plane perpendicular to the $x$ direction, so $\mid\left\langle\varphi_{\mathrm{CBM}}\left|\nabla_{x}\right| \varphi_{\mathrm{VBM}}>\left.\right|^{2}=0\right.$, while for the $y$-direction, no particular symmetry has been observed, that is $\left|<\varphi_{\text {СвM }}\right| \nabla_{y}\left|\varphi_{V \mathrm{VBM}}>\right|^{2} \neq 0$. This lowest-energy optical transition in GeSe results in the high dependence of absorption upon the polarization of incident light near the gap edges $(\sim 920 \mathrm{~nm})$. But optical transitions that favour the $x$-polarized light absorption (e.g., $\mathrm{Vb}_{\mathrm{P} 2} \rightarrow \mathrm{Cb}_{\mathrm{P} 2}$ ) are involved in absorption processes for photon energies above $1.85 \mathrm{eV}\left(\mathrm{Vb}_{\mathrm{M} 1} \rightarrow \mathrm{Cb}_{\mathrm{M} 2}\right)$, which leads to the decrease in the polarization sensitivity. Therefore, the $y$-polarized light absorption continuously covers the range of $1.36-1.85 \mathrm{eV}$ (920-670 $\mathrm{nm}$ in wavelength), while for higher energies, the absorption of $x$-, $y$ - polarized light, even other interband transitions (e.g., $\mathrm{Vb}_{\mathrm{K} 3} \rightarrow \mathrm{Cb}_{\mathrm{K} 3}$ ) will be considered and so the anisotropic absorption may be greatly impacted.

The efficient photoresponse and high dichroic ratio obtained in the above work pave the way for constructing the compact polarimetric imaging system through GeSe prototype device. This device as the photodetector while simultaneously as the polarizer was coupled to the interface board of the proposed system, with functions of sensing both the intensity and polarization of an incident light. And the unpolarized fluorescent lamp used in the above proof of concept imaging system was replaced by the polarized signal source. Here the polarized signal
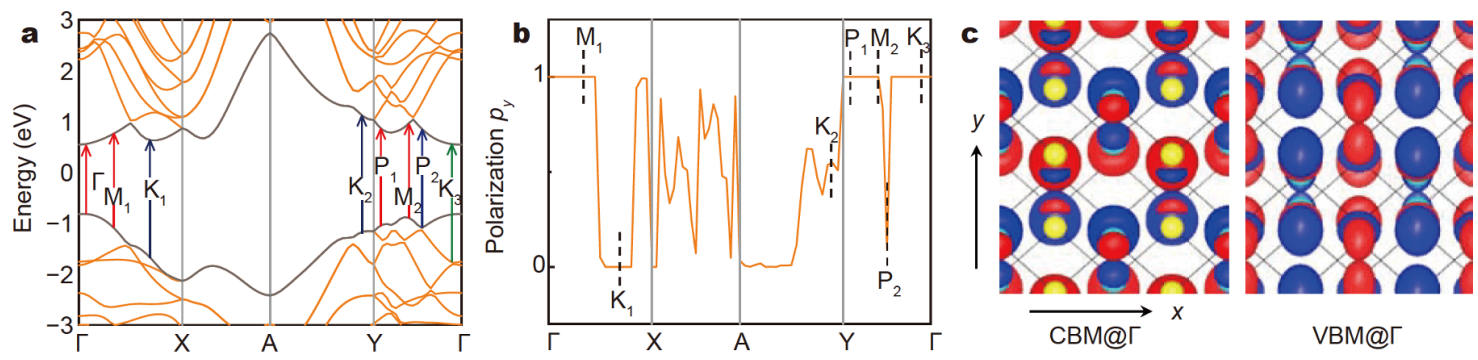

Figure 3 Analysis of optical anisotropy of GeSe based on optical transition amplitudes. (a) Electronic bandstructures for cleaved bulk GeSe obtained from the HSE06 functional, where the CBM and VBM states are marked in grey. The arrowed red (blue) lines represent the possible $y(x)$-polarized light jump. (b) The $p_{y}$ of optical transition $\left|\varphi_{\mathrm{CBM}}>\rightarrow\right| \varphi_{\mathrm{VBM}}>$ along the $k$-points path. (c) The wavefunction contour plots of CBM (left) and VBM (right) states at the $\Gamma$ point. 
source actually comprises of the blackbody-like U-shaped heating coil (also as the detection target), the narrowband pass filter (bandwidth: $\pm 5 \mathrm{~nm}$ ), focusing lens and the polarizing film, as shown in the right panel of Fig. 4a. The radiating signal illuminated onto the GeSe device is nearly linearly polarized. With this re-designed apparatus, the dynamic change of imaging signals with the polarization state of the radiant $\mathrm{U}$-shaped target was obtained through the GeSe-based imaging sensor.

The measurement at the $808 \mathrm{~nm}$ excitation was selected as a representative of the NIR polarimetric imaging, and hence the $808 \mathrm{~nm}$ band-pass filter was equipped in the optical path. For those 3D coloured images (each with $101 \times 76$ pixels) in Fig. 4 b, we used the time-sequential variation of the polarizing film to obtain polarization information from the U-shaped radiation target. And the corresponding contour plots with different incidental polarization were extracted, as shown in Fig. S6. A gradual increase in signal intensity was observed as the polarization angle $(\Delta)$ ranges from $90^{\circ}$ to $0^{\circ}$. Here we have subtracted the background signal from the received radiated information, so that the radiation intensity of tube body for each image can be read out directly. And the contrast ratio of the radiation is about 3.45 with $0^{\circ} / 90^{\circ}$ polarized illumination. The imaging contrast ratio (given by $\left.I_{0^{\circ}} / I_{90^{\circ}}\right)$ used in this paper is positively related to the degree of polarization (here given by $\left(I_{0^{\circ}}-I_{90^{\circ}}\right) /\left(I_{0^{\circ}}+\right.$ $\left.I_{90^{\circ}}\right)$ ). In the literature of polarization imaging, the degree of polarization is commonly used. They both can be used to quantify the degree of incident light signal which is polarized. The similar experiment was implemented under the VIS light excitation (675 and $700 \mathrm{~nm}$ ), and the corresponding contrast ratios of 2.59 and 2.63 were achieved (Fig. S7). This shows that imaging applications based on the 2D GeSe structure are applicable to both VIS and NIR regions. The linearly polarized radiator as the only target in this scene is just a simple example for the research of the potential of 2D GeSe-based polarimetric imaging. This could potentially be applied on more complex scenes, such as different targets or different polarization orientations in a scene.

\section{DISCUSSION}

Most traditional polarimetric imagers are bulky and usually consist of a layer of polarizers and a layer of photo-sensing device. The photo-sensing devices are used for sensing the signal intensity alone. And the multichannel polarizers are combined for obtaining several specified polarization states of incident light from a scene. Such systems are required to extract the polarized signals along four different directions for the calculation of degree of polarization at each pixel.

For anisotropic 2D materials including GeSe, their intrinsic polarized photosensitivity has provided a simplified route for polarimetric imaging. The fabricated device allows for sensing both the signal intensity and the po-
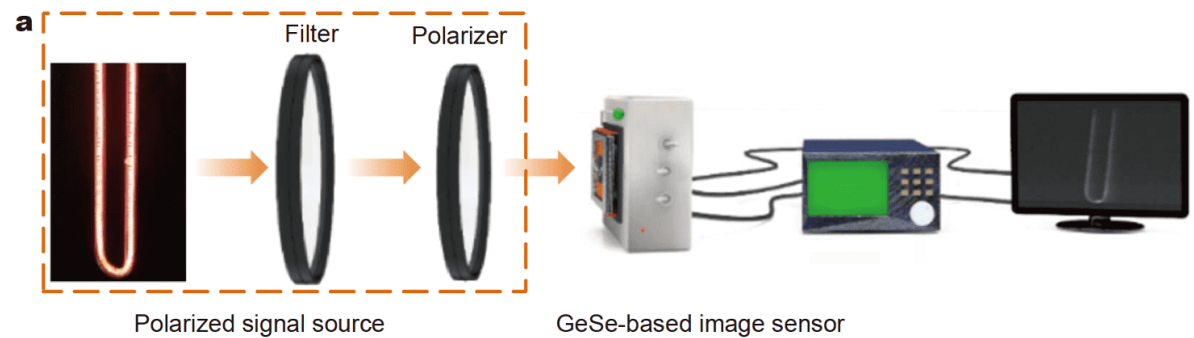

b

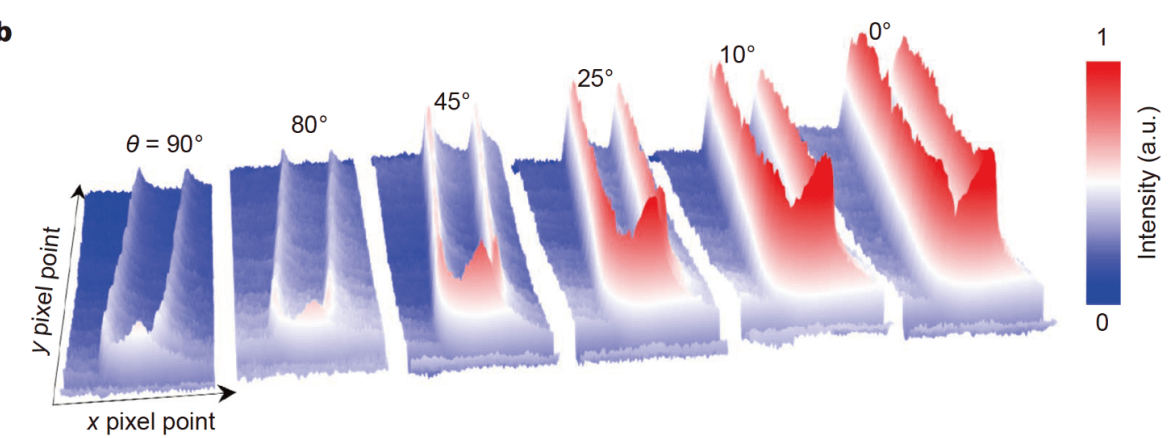

Figure 4 NIR polarimetric imaging of GeSe-based image sensor. (a) Schematic layout of the image scanning system and the photograph of a U-shaped target. (b) With the heated target processed by the infrared filter (CWL: $808 \mathrm{~nm}$ ), the 3D imaging results at an applied voltage of $0.65 \mathrm{~V}$ with the excitation polarization $\left(90^{\circ}, 80^{\circ}, 45^{\circ}, 25^{\circ}, 10^{\circ}, 0^{\circ}\right.$ from left to right panels). 
larization states of incident light from a scene. Thus, the polarizer is not required in the optical system. The PC along different polarized directions can readily be attained by rotating the GeSe device for the calculation of degree of polarization at each pixel. In addition, the polarization extinction ratio can be further enhanced for longer wavelength, such as 3.9 for $940 \mathrm{~nm}$ illumination, according to the above polarization-sensitive PC studies. This makes GeSe a potential material for NIR polarized imaging devices to reach a higher degree of polarization.

\section{CONCLUSIONS}

In summary, we have fabricated and characterized the $\mathrm{Au}-\mathrm{GeSe}-\mathrm{Au}$ device, as well as exploited the intrinsic inplane anisotropy of this material for the realization of functionality relating with polarization-sensitive photodetector/imager in the VIS-NIR spectral regime. This device demonstrated here runs in the photoconductive mode and exhibits highly sensitive spectral $R_{\mathrm{ex}}$ of about $10^{3} \mathrm{~A} \mathrm{~W}^{-1}$ at a very low power, i.e., $\mu \mathrm{W}$ level. And the response with rise/decay time of $22.7 / 49.5 \mu$ at $830 \mathrm{~nm}$ makes these nanodevices suitable for fast imaging. In addition, the layered GeSe possesses distinct polarizationsensitive spectral response in VIS-NIR band. To fully exploit the above advantages of GeSe, we have integrated this $2 \mathrm{D}$ GeSe device into the image sensor for the polarization imaging. The polarization information of the detection target was extracted with the contrast ratio of $2.59,2.63,3.45$ in the VIS-NIR spectral bands $(675,700$, $808 \mathrm{~nm})$. The proposed imager in this study reveals the ability to sense dual-band polarization signals and opens up new prospects for $2 \mathrm{D}$ material-based multiband polarization imaging system, focal plane polarimeters, and even bioimaging.

Received 17 August 2020; accepted 30 September 2020; published online 24 December 2020

1 Rubin NA, D'Aversa G, Chevalier P, et al. Matrix Fourier optics enables a compact full-Stokes polarization camera. Science, 2019, 365: eaax 1839

2 Empedocles SA, Neuhauser R, Bawendi MG. Three-dimensional orientation measurements of symmetric single chromophores using polarization microscopy. Nature, 1999, 399: 126-130

3 Li Q, Li ZF, Li N, et al. High-polarization-discriminating infrared detection using a single quantum well sandwiched in plasmonic micro-cavity. Sci Rep, 2014, 4: 6332

4 Niu S, Joe G, Zhao H, et al. Giant optical anisotropy in a quasione-dimensional crystal. Nat Photon, 2018, 12: 392-396

5 Zheng F, Tao X, Yang M, et al. Design of efficient superconducting nanowire single photon detectors with high polarization sensitivity for polarimetric imaging. J Opt Soc Am B, 2016, 33: 2256-2264
6 Abrahamsson S, McQuilken M, Mehta SB, et al. Multifocus polarization microscope (MF-PolScope) for 3D polarization imaging of up to 25 focal planes simultaneously. Opt Express, 2015, 23: 7734-7754

7 Zhao Y, Peng Q, Yi C, et al. Multiband polarization imaging. J Sens, 2016, 2016: 5985673

8 Tyo JS, Goldstein DL, Chenault DB, et al. Review of passive imaging polarimetry for remote sensing applications. Appl Opt, 2006, 45: 5453-5469

9 Laux E, Genet C, Skauli T, et al. Plasmonic photon sorters for spectral and polarimetric imaging. Nat Photon, 2008, 2: 161-164

10 Nayar SK, Fang XS, Boult T. Separation of reflection components using color and polarization. Int J Comput Vision, 1997, 21: 163186

11 Mohammadi E, Behdad N. A wide dynamic range polarization sensing long wave infrared detector. Sci Rep, 2017, 7: 17475

12 Kippelen B, Marder SR, Hendrickx E, et al. Infrared photorefractive polymers and their applications for imaging. Science, 1998, 279: $54-57$

13 Kulkarni M, Gruev V. Integrated spectral-polarization imaging sensor with aluminum nanowire polarization filters. Opt Express, 2012, 20: 22997-23012

14 Nanot S, Cummings AW, Pint CL, et al. Broadband, polarizationsensitive photodetector based on optically-thick films of macroscopically long, dense and aligned carbon nanotubes. Sci Rep, 2013, 3: 1335

15 Goossens S, Navickaite G, Monasterio C, et al. Broadband image sensor array based on grapheme-CMOS integration. Nat Photon, 2017, 11: 366-371

16 Guo N, Hu W, Jiang T, et al. High-quality infrared imaging with graphene photodetectors at room temperature. Nanoscale, 2016, 8: 16065-16072

17 Lee YT, Jeon PJ, Han JH, et al. Mixed-dimensional 1D ZnO-2D $\mathrm{WSe}_{2}$ van der Waals heterojunction device for photosensors. Adv Funct Mater, 2017, 27: 1703822

18 Engel M, Steiner M, Avouris P. Black phosphorus photodetector for multispectral, high-resolution imaging. Nano Lett, 2014, 14: 6414-6417

19 Yin J, Tan Z, Hong H, et al. Ultrafast and highly sensitive infrared photodetectors based on two-dimensional oxyselenide crystals. Nat Commun, 2018, 9: 3311

20 Mennel L, Symonowicz J, Wachter S, et al. Ultrafast machine vision with 2D material neural network image sensors. Nature, 2020, 579: 62-66

21 Yuan H, Liu X, Afshinmanesh F, et al. Polarization-sensitive broadband photodetector using a black phosphorus vertical p-n junction. Nat Nanotech, 2015, 10: 707-713

22 Bullock J, Amani M, Cho J, et al. Polarization-resolved black phosphorus/molybdenum disulfide mid-wave infrared photodiodes with high detectivity at room temperature. Nat Photon, 2018, 12: 601-607

23 Yang Y, Liu SC, Yang W, et al. Air-stable in-plane anisotropic $\mathrm{GeSe}_{2}$ for highly polarization-sensitive photodetection in short wave region. J Am Chem Soc, 2018, 140: 4150-4156

24 Xia F, Wang H, Jia Y. Rediscovering black phosphorus as an anisotropic layered material for optoelectronics and electronics. Nat Commun, 2014, 5: 4458

25 Mukherjee B, Cai Y, Tan HR, et al. NIR Schottky photodetectors based on individual single-crystalline GeSe nanosheet. ACS Appl Mater Interfaces, 2013, 5: 9594-9604 
Zhou X, Hu X, Jin B, et al. Highly anisotropic GeSe nanosheets for phototransistors with ultrahigh photoresponsivity. Adv Sci, 2018, 5: 1800478

27 von Rohr FO, Ji H, Cevallos FA, et al. High-pressure synthesis and characterization of $\beta$-GeSe: A six-membered-ring semiconductor in an uncommon boat conformation. J Am Chem Soc, 2017, 139: 2771-2777

28 Xue DJ, Liu SC, Dai CM, et al. GeSe thin-film solar cells fabricated by self-regulated rapid thermal sublimation. J Am Chem Soc, 2017, 139: 958-965

29 Ye Y, Guo Q, Liu X, et al. Two-dimensional GeSe as an isostructural and isoelectronic analogue of phosphorene: Sonicationassisted synthesis, chemical stability, and optical properties. Chem Mater, 2017, 29: 8361-8368

30 Guo Q, Pospischil A, Bhuiyan M, et al. Black phosphorus midinfrared photodetectors with high gain. Nano Lett, 2016, 16: 46484655

31 Wu D, Ma Y, Niu Y, et al. Ultrabroadband photosensitivity from visible to terahertz at room temperature. Sci Adv, 2018, 4: eaao3057

32 Cheng R, Wang F, Yin L, et al. High-performance, multifunctional devices based on asymmetric van der Waals heterostructures. Nat Electron, 2018, 1: 356-361

33 Wang $\mathrm{P}$, Liu S, Luo W, et al. Arrayed van der Waals broadband detectors for dual-band detection. Adv Mater, 2017, 29: 1604439

34 Yuan X, Tang L, Liu S, et al. Arrayed van der Waals vertical heterostructures based on 2D GaSe grown by molecular beam epitaxy. Nano Lett, 2015, 15: 3571-3577

35 Hwang DK, Lee YT, Lee HS, et al. Ultrasensitive PbS quantumdot-sensitized InGaZnO hybrid photoinverter for near-infrared detection and imaging with high photogain. NPG Asia Mater, 2016, 8: e233

36 Wang X, Li Y, Huang L, et al. Short-wave near-infrared linear dichroism of two-dimensional germanium selenide. J Am Chem Soc, 2017, 139: 14976-14982

37 Zhang E, Wang P, Li Z, et al. Tunable ambipolar polarizationsensitive photodetectors based on high-anisotropy $\mathrm{ReSe}_{2}$ nanosheets. ACS Nano, 2016, 10: 8067-8077

38 Buscema M, Island JO, Groenendijk DJ, et al. Photocurrent generation with two-dimensional van der Waals semiconductors. Chem Soc Rev, 2015, 44: 3691-3718

39 Long M, Wang P, Fang H, et al. Progress, challenges, and opportunities for $2 \mathrm{D}$ material based photodetectors. Adv Funct Mater, 2019, 29: 1803807

40 Léonard F, Talin AA. Electrical contacts to one- and two-dimensional nanomaterials. Nat Nanotech, 2011, 6: 773-783

41 Allain A, Kang J, Banerjee $\mathrm{K}$, et al. Electrical contacts to twodimensional semiconductors. Nat Mater, 2015, 14: 1195-1205

Acknowledgements This work was supported by the National Natural Science Foundation of China (61622406, 61904015, 11674310, 61725505 and 11734016), the National Key Research and Development Program of China (2017YFA0207500), the Strategic Priority Research Program of Chinese Academy of Sciences (XDB30000000), the “The Pearl River Talent Recruitment Program" (2019ZT08X639), and Beijing National Laboratory for Molecular Sciences (BNLMS201908).

Author contributions $\mathrm{Wei} \mathrm{Z}$ and $\mathrm{Hu} \mathrm{W}$ supervised this project and designed the experiments; Wang $\mathrm{X}$ and Zhong $\mathrm{F}$ performed sample fabrication and optical measurements; Kang J and Pan L carried out the theoretical part; Wang X, Liu C, Lei M, Wang F, Zhou Z, Cui Y, Liu K, Wang J, Shen G, Shan C and Li J performed the data analysis and interpretation; Wei $\mathrm{Z}$ and Wang $\mathrm{X}$ wrote the paper, with input from all authors. All authors contributed to the general discussion.

Conflict of interest The authors declare that they have no conflict of interest.

Supplementary information Experimental details and supporting data are available in the online version of the paper.

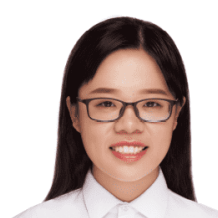

Xiaoting Wang received her $\mathrm{PhD}$ degree in 2018 from the Institute of Semiconductors, Chinese Academy of Sciences. Then she joined Beijing University of Posts and Telecommunications and has been working as a postdoctoral fellow. Her research group focuses on the syntheses of $2 \mathrm{D}$ layered materials and their related electronic and photoelectric properties.

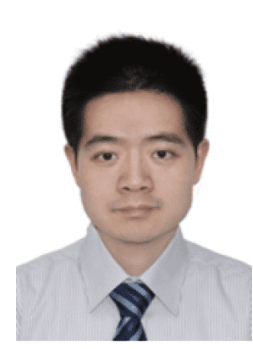

Zhongming Wei received his $\mathrm{PhD}$ degree in 2010 from the Institute of Chemistry, Chinese Academy of Sciences under the supervision of Prof. Daoben Zhu and Prof. Wei Xu. From 2010 to 2015 , he worked as a postdoctoral fellow and then as assistant professor in Prof. Thomas Bjørnholm's group at the University of Copenhagen, Denmark. Currently, he is working as a professor at the Institute of Semiconductors, Chinese Academy of Sciences. His research interests include low-dimensional nanostructured materials and their (opto)electronic devices.

\section{基于各向异性二维GeSe的无偏振器偏振图像传感器}

王晓亭 ${ }^{1 \dagger}$, 仲方 ${ }^{2 \dagger}$, 康俊 ${ }^{3}$, 刘灿 ${ }^{4}$, 雷鸣 ${ }^{5}$, 潘龙飞 ${ }^{1}$, 王海露 ${ }^{2}$, 王芳 ${ }^{2}$, 周子琦, 崔宇 ${ }^{1}$, 刘开辉, 王建禄 ${ }^{2}$, 沈国震, 单崇新 ${ }^{6}$, 李京波 ${ }^{7}$, 胡伟达 ${ }^{2^{*}}$, 魏钟鸣 ${ }^{1^{*}}$

摘要 偏振是光的一个重要信息, 偏振探测可以把信息量从三维 (光强、光谱和空间)扩充到七维(光强、光谱、空间、偏振度、光 偏振等), 为成像物体提供关键的视觉信息(如表面粗粘度、几何形 状或方向), 因此偏振成像技术在目标检测等领域有着巨大的潜力. 然而这些领域往往需要复杂的偏振编码, 现有的复杂透镜系统和 偏振器限制了集成成像传感器的小型化能力. 本文通过二维各向 异性 $\alpha-G e S e$ 半导体, 成功实现了无偏振器的偏振敏感可见-近红外 光电探测器/成像仪. 作为传感器系统的关键部件, 该原型 $\mathrm{Au}$ / $\mathrm{GeSe} / \mathrm{Au}$ 光电探测器具有灵敏度高、光谱响应宽、响应速度快 $\left(\sim 10^{3} \mathrm{~A} \mathrm{~W}^{-1}, 400-1050 \mathrm{~nm}, 22.7 / 49.5 \mu \mathrm{s}\right)$ 等优点. 此外, 该器件在 690-1050 nm 光谱范围内表现出独特的偏振灵敏度, 并且对沿 $y$ 方 向的偏振光吸收最强, 这一点通过分析 $\alpha-G e S e$ 的光跃迁行为也得 到了证实. 最后, 将 $2 \mathrm{D}-\mathrm{GeSe}$ 器件应用到成像系统中进行偏振成像, 在 $808 \mathrm{~nm}$ 近红外波段处, 在不同的偏振方向上, 辐射目标的对比度 为3.45. 这种成像仪在没有偏振器的情况下, 能够在场景中感知双 频偏振信号, 为偏振成像传感器阵列的广泛应用奠定了基础. 